\section{6}

Department of Hematology, Tokyo Medical University, Tokyo, Japan

${ }^{2}$ Department of Pathology, Tokai University, Isehara, Japan

\section{Correspondence to}

Dr Yuu Saitoh, Department of Hematology, Tokyo Medical University, 6-7-1 Nishi-shinjuku, Shinjuku-ku, Tokyo 160-0023, Japan; yusaitoh@tokyo-med. ac.jp

Received 4 April 2017 Revised 25 May 2017 Accepted 29 May 2017 Published Online First 14 July 2017

\section{CrossMark}

\title{
Unique radiological features of two cases of primary pulmonary diffuse large B-cell lymphoma
}

\author{
Yuu Saitoh, ${ }^{1}$ Ayae Ohnishi-Amemiya, ${ }^{1}$ Michiyo Asano, ${ }^{1}$ Yuko Tanaka, ${ }^{1}$ \\ Seiichiro Yoshizawa, ${ }^{1}$ Hiroaki Fujimoto, ${ }^{1}$ Yoshikazu Itoh, ${ }^{1}$ Naoya Nakamura, ${ }^{2}$ \\ Kazuma Ohyashiki ${ }^{1}$
}

\section{INTRODUCTION}

Radiographic infiltration of the lungs by malignant lymphoma is often observed, with a reported frequency of approximately $25 \%$. On the other hand, primary pulmonary lymphoma (PPL) is very rare. According to previous reports, PPL was observed in only $3 \%$ of patients with extranodal lymphoma, and in less than $1 \%$ of patients with non-Hodgkin's lymphoma. Most cases of PPL (58\%-87\%) are marginal zone lymphomas of the mucosa-associated lymphoid tissue (MALT) type. ${ }^{1}$ However, primary lung diffuse large B-cell lymphoma (DLBCL) is rare $(10 \%) .^{2}$ Here we present two cases of primary lung DLBCL displaying unique imaging.

\section{CASE REPORT}

\section{Case 1}

A 56-year-old woman complaining of dyspnoea and fever was admitted to our hospital. Consolidation in the bilateral upper fields on chest radiological examination and multiple pulmonary nodules on CT were the only abnormalities detected by radiography (Figure 1A).

Upper and lower endoscopy showed no evidence of gastrointestinal cancer. A trans-bronchial lung biopsy (TBLB) was subsequently performed for histopathological analysis. Haematoxylin and eosin staining of tissue sections showed diffuse and monotonous proliferation of large round cells, in the form of sheets within interstitial lung tissue (Figure 1B). Immunohistochemical staining demonstrated that the tumour cells were positive for CD5 and CD20, but negative for CD3 and cytokeratin . A high Ki-67 proliferation index (>90\%) was also found. The diagnosis was CD5-positive DLBCL accompanied by an intravascular lymphoma-like lesion. After urgent admission, gallium-67 scintigraphy was performed instead of positron emission tomography due to its availability in our institute. It demonstrated multiple lesions in the bilateral upper lung fields without any other lesions (Figure 1C). There was no involvement of either the central nervous system (CNS) or the bone marrow. Therefore, the patient was diagnosed with PPL. ${ }^{1}$ She was treated with a rituximab, cyclophosphamide, doxorubicin, vincristine and prednisolone (R-CHOP) regimen. After eight courses of R-CHOP therapy, the patient achieved complete remission, which has been maintained to date without relapse in the CNS, despite having CD5-positive DLBCL.

\section{Case 2}

A 77-year-old man presented with dyspnoea. A chest CT displayed multiple nodular lesions in bilateral lung fields (Figure 1D). Histopathological analysis of TBLB demonstrated diffuse and monotonous proliferation of large round cells (Figure 1E). Immunohistochemical staining showed that the tumour cells were positive for CD20 but negative for CD3, CD5 and cytokeratin. A high Ki-67 proliferation index (>90\%) was also found. All findings were compatible with DLBCL. As in case 1, we performed gallium-67 scintigraphy (Figure $1 \mathrm{~F})$. As bone marrow biopsy showed no apparent abnormalities, the patient was diagnosed with PPL. ${ }^{1}$ Fluorescence in situ hybridization analysis for the presence of the API2-MALT1 fusion gene in lung tissue was performed using a nodular lesion that had been biopsied 2 years previously. However, we were unable to confirm MALT lymphoma as a pre-existing disease. Therefore, the patient was diagnosed with de novo DLBCL. He was treated with R-CHOP therapy and achieved complete remission which has been maintained to date.

\section{DISCUSSION}

The diagnostic criteria for primary lung DLBCL include pathological and immunohistochemical features of DLBCL, a primary lesion restricted to the lung (parenchyma and/or bronchi) with or without minimal hilar lymph node involvement, and clinical, radiological and pathological exclusion of disease at distant sites. ${ }^{3}$ Both of our cases met these criteria.

Chest CT findings are variable in PPL and include solitary or multiple pulmonary nodules, masses, consolidation, hilar/mediastinal adenopathy, pleural effusion and, rarely, direct chest wall invasion. In particular, primary pulmonary DLBCL should be suspected in patients when masses or mass-like areas of consolidation and pulmonary nodules are identified together with air bronchograms and a halo of ground-glass shadowing at lesion margins. However, to our knowledge there are no reports to date on the imaging finding of patients with primary DLBCL. Many previous reports ${ }^{45}$ have demonstrated the presence of a solitary nodular lesion in DLBCL. Multiple heterogeneous lesions in the lung are usually seen in patients with primary carcinoma or metastatic cancer. In our first case, nodular lesions were scattered in the bilateral upper lung fields. To our knowledge, no 

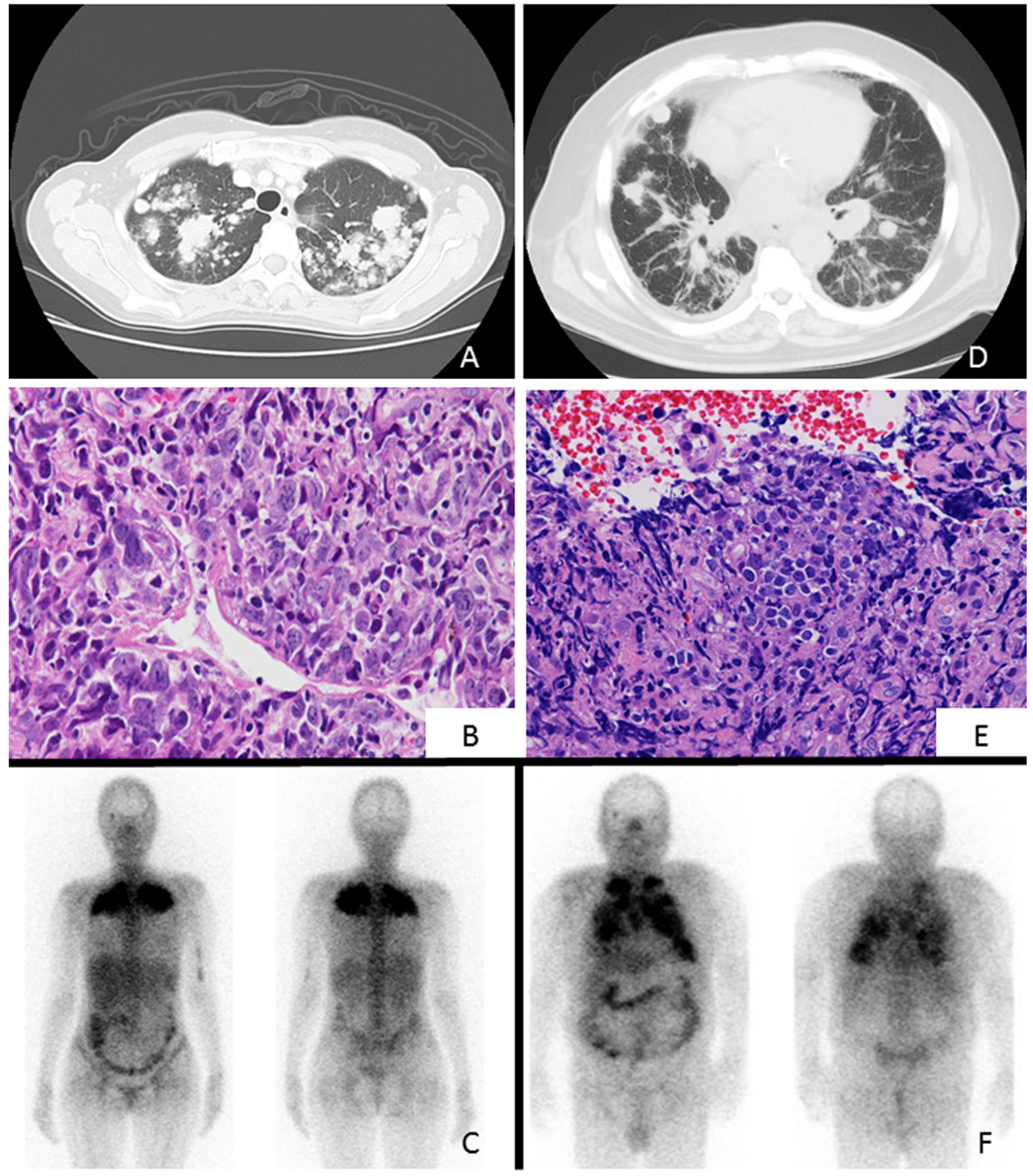

Figure 1 Radiographical and histological findings. (A) Case 1: chest CT showing multiple pulmonary nodules. (B) Case 1: haematoxylin-eosin staining showing diffuse prolifaration of large cells. (C) Case 1: gallium-67 scintigraphy showing multiple lesion int the bilateral upper lung fields. (D) Case 2: chest CT showing multile pulmonary nodules. (E) Case 2: haematoxylin-eosin staining shohing diffuse and monotonus prolifaration of large cell . (F) Case 2: gallium-67 scintigraphy showing multiple lesion in the bilateral lung fieds.

cases have been reported to date displaying such radiological features. The key imaging finding of this patient was intratumoral bronchial translucency. As this is not typical in metastatic pulmonary tumours, lymphoma was suspected and histological analysis was promptly performed.

In summary, primary lung DLBCL is a very rare disease. In our present cases, only multiple nodular lesions in the lung were observed at the time of diagnosis and CT examination displayed atypical images. Therefore, in such cases, the possibility of malignant lymphoma should be considered and thorough histological analysis should be performed.

Contributors YS treated patients, and wrote the initial draft of the manuscript. KO and YT contributed to assist in the preparation of the manuscript. All other authors have contributed to treat patiens and interpretation, and critically reviewed the manuscript. The final version of the manuscript was approved by all authors.

Competing interests $\mathrm{KO}$ received grants from Chugai pharma, Novartis pharma KK, Bristol-Myers Squibb, Celegen, Jansen pharma, and personal fees from Novartis pharma, Bristol-Myers Squibb, Celegen, Nippon Shinyaku, Dainippon Sumitomo,
Fujirebio, Phizer, Jansen pharma, Taiho pharma, Otsuka pharma, Alexion, MSD, Kyowa Hakko Kirin, outside the submitted work.

\section{Patient consent Obtained.}

Provenance and peer review Not commissioned; externally peer reviewed.

(c) Article author(s) (or their employer(s) unless otherwise stated in the text of the article) 2017. All rights reserved. No commercial use is permitted unless otherwise expressly granted.

\section{REFERENCES}

1 Cadranel J, Wislez M, Antoine M. Primary pulmonary lymphoma. Eur Respir J 2002:20:750-62.

2 Zinzani PL, Martelli M, Poletti V, et al. A project of the Italian Society of Hematology, the Italian Society of Experimental Hematology and the Italian Group for Bone Marrow Transplantation. Hematologica 2008;93:1364-71.

3 Wróbel T, Dzietczenia J, Prochorec-Sobieszek M, et al. Primary pulmonary diffuse large B-cell lymphoma. Am J Hematol 2012;87:107-8.

4 Jiang AG, Gao XY, Lu HY. Diagnosis and management of a patient with primary pulmonary diffuse large B-cell lymphoma: a case report and review of the literature. Exp Ther Med 2014;8:797-800.

$5 \mathrm{Xu} \mathrm{H}, \mathrm{Xu} \mathrm{K}$, Wang R, et al. Primary pulmonary diffuse large B-cell lymphoma on FDG PET/CT-MRI and DWI. Medicine 2015;94:e1210. 